University of Wollongong

Research Online

Faculty of Engineering and Information

Faculty of Engineering and Information

Sciences - Papers: Part B

Sciences

2018

Behavior of Steel Furnace Slag, Coal Wash, and Rubber Crumb Mixtures with Special Relevance to Stress-Dilatancy Relation

Yujie Qi

University of Wollongong, qyujie@uow.edu.au

Buddhima Indraratna

University of Wollongong, indra@uow.edu.au

J S. Vinod

University of Wollongong, vinod@uow.edu.au

Follow this and additional works at: https://ro.uow.edu.au/eispapers1

Part of the Engineering Commons, and the Science and Technology Studies Commons

Research Online is the open access institutional repository for the University of Wollongong. For further information contact the UOW Library: research-pubs@uow.edu.au 


\title{
Behavior of Steel Furnace Slag, Coal Wash, and Rubber Crumb Mixtures with Special Relevance to Stress-Dilatancy Relation
}

\author{
Abstract \\ The influence of rubber crumbs on the dilatancy behavior and critical state of steel furnace slag (SFS), \\ coal wash (CW), and rubber crumbs (RC) mixtures was investigated via a series of monotonic drained \\ triaxial tests. These tests revealed that $\mathrm{RC}$ contents $\left(\mathrm{R}_{\mathrm{b}}, \%\right)$ have a significant influence on the dilatancy \\ behavior and critical state of the aforementioned waste mixtures; in fact, as more $\mathrm{R}_{\mathrm{b}}$ is added, dilatancy \\ and the slope of the critical state line in e-In p' space decreases. Within the framework of critical state \\ soil mechanics, a dilatancy model for SFS+CW+RC mixtures was proposed and validated using \\ experimental data. This model also captured the energy-absorbing property of RC using an empirical \\ relationship between the total work input $\mathrm{W}_{\text {total }}$ and the critical stress ratio $\mathrm{M}_{\mathrm{cs}}$. \\ Disciplines \\ Engineering | Science and Technology Studies \\ Publication Details \\ Qi, Y., Indraratna, B. \& Vinod, J. S. (2018). Behavior of Steel Furnace Slag, Coal Wash, and Rubber Crumb \\ Mixtures with Special Relevance to Stress-Dilatancy Relation. Journal Of Materials In Civil Engineering, 30 \\ (11), 04018276-1-04018276-10.
}


Behavior of Steel Furnace Slag, Coal Wash, and Rubber Crumb Mixtures, with Special Relevance to Stress-dilatancy Relation

\section{Yujie Qi}

$\mathrm{PhD}, \mathrm{Ms}, \mathrm{Bs}$

Research Associate, Centre for Geomechanics and Railway Engineering, ARC Industry Transformation Training Centre for Advanced Rail Track Technologies, Faculty of Engineering, University of Wollongong, Wollongong, NSW 2522, Australia

\section{Buddhima Indraratna}

PhD (Alberta), MSc and BSc-Hons (London), FTSE, FIEAust, FASCE, FGS, FAusIMM, FIES, DIC, CEng, CPEng

Distinguished Professor, Research Director and Foundation Director, Civil Engineering, Centre for Geomechanics and Railway Engineering, ARC Industry Transformation Training Centre for Advanced Rail Track Technologies, Faculty of Engineering, University of Wollongong, Wollongong, NSW 2522, Australia

\section{Jayan S. Vinod}

$\mathrm{PhD}$, M.Tech. B.Tech.

Associate Professor, Centre for Geomechanics and Railway Engineering, ARC Industry Transformation Training Centre for Advanced Rail Track Technologies, Faculty of Engineering and Information Sciences, University of Wollongong, Wollongong, NSW 2522, Australia

$\dagger$ Author for correspondence:

Dr Jayan S. Vinod

School of Civil, Mining, and Environmental Engineering,

Faculty of Engineering and Information Sciences, University of Wollongong Wollongong, NSW 2522

AUSTRALIA

Ph: +61 0242214089

Email: vinod@uow.edu.au

Published by: Journal of Materials in Civil Engineering 
37 Abstract: The influence of rubber crumbs on the dilatancy behavior and critical state of 38 SFS $+\mathrm{CW}+\mathrm{RC}$ mixtures (i.e., blends of steel furnace slag, coal wash, and rubber crumbs) has 39 been investigated via a series of monotonic drained triaxial tests. These tests reveal that $\mathrm{RC}$ 40 contents $\left(R_{b}, \%\right)$ have a significant influence on the dilatancy behavior and critical state of 41 the aforementioned waste mixtures; in fact as more $R_{b}$ is added, dilatancy and the slope of 42 the critical state line in $e-\ln p^{\prime}$ space decreases. Within the framework of critical state soil 43 mechanics, a dilatancy model for SFS+CW+RC mixtures has been proposed and validated 44 using experimental data. This model also captured the energy absorbing property of RC using 45 an empirical relationship between the total work input $W_{\text {total }}$ and the critical stress ratio $M_{c s}$.

46 KEYWORDS: Steel furnace slag; coal wash; rubber crumbs; critical state; energy absorbing 47 property; dilatancy 
49 Steel furnace slag (SFS) and coal wash (CW) are waste by-products of steel making and coal mining, whereas rubber crumbs $(\mathrm{RC})$ are derived from waste tires, and since they occupy large amounts of useable land their long term effect on the environment is extremely detrimental. One of the best ways of dealing with these materials is to recycle them into geotechnical projects such as port reclamations, where different blends of SFS+CW have already been used successfully (Chiaro et al., 2013). However, while incorporating RC into $\mathrm{SFS}+\mathrm{CW}$ blends can further reduce the particle breakage of $\mathrm{CW}$ and swelling of SFS, as well as increasing the energy absorbing capacity of these waste mixtures, a better understanding of the effect that $\mathrm{RC}$ has on the geotechnical behavior of $\mathrm{SFS}+\mathrm{CW}+\mathrm{RC}$ mixtures from a mathematical perspective is urgently needed. Despite the research already carried out to investigate the behavior of soil-rubber mixtures in the laboratory, only a few focused on the theoretical models used to predict the behavior of soil-rubber mixtures.

Stress-dilatancy is a fundamental aspect needed to model the stress-strain behavior of soil. It has been suggested that dilatancy in its initial form is a unique function with the stress ratio $\eta$ (Taylor, 1948), but granular materials differ from cohesive soils, so dilatancy not only depends on $\eta$ but also on the density and stress history of soil (e.g. Rowe, 1962; Nova and Wood, 1979). To avoid using too many parameters for a single granular material with different initial conditions, Been and Jefferies (1985) introduced a state parameter $\psi$ based on a critical state concept which relates the density and stress history of soil. Until now $\psi$ and the critical state concept has been widely used to model the dilatancy of granular materials (e.g. Wan and Guo, 1998; Li and Dafalias, 2000; Wang et al., 1990); more recently they have also been successfully extended to tire chips/shreds - sand mixtures (e.g. Mashiri et al., 2015;

71 Youwai and Bergado, 2003). 
However, only limited studies have been carried out on the dilatancy behavior of tire soil mixtures (e.g. Mashiri et al. 2015). Mashiri et al. (2015) has developed a dilatancy model for sand-tire chip mixtures. It is to be noted that currently there is no or very limited literature available on the dilatancy behavior of waste mixture -tire crumb mixtures. Rubber crumbs behave differently with tire chips. Tire chips are relatively larger pieces of tire derived aggregate (typically the size of a gravel) that may also include reinforcing elements such as polymeric fibres (of various types) and/or steel fibres from remaining wire reinforcement (Mashiri et al., 2017; Fu et al., 2017). Thus, tire chips look and behave more like a composite material whereas rubber crumbs is made of (and behave more like) a single material (i.e., just rubber). As a result, these two are inherently different materials with corresponding mechanical behavior reflecting their original constituents (i.e., rubber crumbs is mainly isotropic whereas tire chips may behave anisotropically due to the presence of any reinforcing inclusions still present in the chips) (Mashiri et al., 2017). Therefore, previous investigations on tire chips/shreds-soil mixtures cannot totally reflect the behavior of RCwaste mixtures.

Moreover, no previous studies have incorporated the energy absorbing property of RC in modelling rubber-soil materials. As energy absorbing capacity is a very important geotechnical property of rubber materials, it is important to incorporate its influence when modelling the behavior of RC-waste mixtures. Therefore, in this study the dilatancy behavior of $\mathrm{SFS}+\mathrm{CW}+\mathrm{RC}$ mixtures was investigated based on a series of monotonic drained triaxial tests. The critical state and the energy absorbing property of the mixtures were also examined.

\section{Materials and Methodology}

The coal wash (specific gravity $\left.G_{s}=2.11\right)$ and steel furnace slag $\left(G_{s}=3.43\right)$ used in this study are from Illawarra Coal and Australia Steel Milling Services, respectively. The 
granulated $\mathrm{RC}\left(G_{s}=1.15\right)$ from waste tires are in three different size $(0-2.3 \mathrm{~mm}, 0.3-3 \mathrm{~mm}$, and 1-7 mm); the appearance of these waste materials can be seen in Fig.1, and the particle size distribution (PSD) curves of SFS, CW, and RC are shown in Fig.2. Form Fig.1, it can be seen that the $\mathrm{CW}$ aggregates are composed of both angular and relatively flaky grains, $\mathrm{RC}$ are dark angular granulated particles, while SFS is grey and tends to be a bit round. Based on the unified soil classification system, SFS and CW are classified as well-graded gravel with siltysand, and well-graded sand with gravel, respectively.

As gradation can influence the behavior of soils (Salvatore et al., 2015), all the mixtures tested in this study are mixed to the same gradation (the target PSD) shown in Fig.2. The blending ratio of SFS and CW is 7:3 (by weight) because with this ratio the SFS+CW blends have a relatively low particle breakage and swelling potential while maintaining a high shear strength (Indraratna et al., 2017). The RC contents are 0\%, 10\%, 20\%, 30\%, 40\% by weight; every specimen was prepared with the optimum moisture content and then compacted to achieve an initial dry unit weight equal to $95 \%$ of their maximum dry density. To achieve the target particle size distribution (PSD), the preparation method followed a previous study by Tasalloti et al. (2015). The waste materials were sieved and separated into different particle sizes. For each SFS+CW+RC mixture, the dry mass required of each particle size was backcalculated based on the target PSD curve, and then the exact mass corresponding to a given size range was weighed and mixed thoroughly to obtain a uniform blend.

After the dried mixtures were prepared, the required amount of water was added to the mixture, and the mixture was blended thoroughly. The triaxial test specimen $(50 \mathrm{~mm}$ in diameter and $100 \mathrm{~mm}$ high) was then prepared and compacted using a split mould. Each specimen was compacted in 5 layers using a drop hammer. Before placing the subsequent layer, the previous layer was roughened to avoid any layering during the shearing. 

three stages, i.e. saturation, consolidation, and shearing. During saturation stage, the air was firstly expelled by flooding the specimen(s) from the bottom with de-aired water, and then the specimen was saturated with back pressure until the Skempton's B-value $>0.98$. Isotropic consolidation was then applied with an effective confining pressure of ${\sigma^{\prime}}_{3}(10,40$ or $70 \mathrm{kPa})$. After consolidation, the specimen was sheared under drained conditions at a constant strain rate of $0.2 \mathrm{~mm} / \mathrm{min}$ until $25 \%$ axial strain was reached. For each $\mathrm{RC}$ content, at the same test conditions, at least two replicated tests have been done. For instance, a replicated test for SFS+CW+RC mixtures with $40 \%$ RC has been shown in Fig.3 (c) and (f). It is evident that the replicated test closely predicts the same (original test) behavior of $\mathrm{SFS}+\mathrm{CW}+\mathrm{RC}$ mixtures with $40 \% \mathrm{RC}$ at $\sigma_{3}^{\prime}=70 \mathrm{kPa}$. The test number, dry density of each sample, as well as the initial void ratio after consolidation (before shearing) are shown in Table 1.

\section{Experimental Results and Analysis}

\section{Stress-strain behavior}

Fig.3 shows the typical stress-strain curves for SFS+CW+RC mixtures with different $R_{b}(\%)$ at different confining pressures, i.e. $\sigma_{3}^{\prime}=10,40$, and $70 \mathrm{kPa}$. From Fig.3(a-c) it can be observed that the peak deviator stress $q_{\text {peak }}$ increases as $\sigma_{3}^{\prime}$ increases, but it decreases as $R_{b}$ (\%) increases indicating the low shear strength of rubber crumbs. As more RC is included, the axial strain corresponding to $q_{\text {peak }}$ increases as the specimen changes from brittle to ductile. This may be attributed to the increase of rubber-to-rubber interaction in SFS+CW+RC mixtures (Sheikh et al., 2013). Moreover, all the specimens exhibited a predominantly strain softening behavior accompanied by a contractive-dilative response. expected, the waste mixtures become much more contractive as $R_{b}(\%)$ increases because of 
the high compressibility of rubber materials. Under the effective confining pressure $\sigma_{3}^{\prime}=$ 10,40 , and $70 \mathrm{kPa}$, the volumetric strain of specimens with $R_{b} \leq 10 \%$ all reached a stable state before the end of the test, whereas those with $R_{b} \geq 20 \%$ still keep dilation by the end of 147 the test.

\section{Dilatancy behavior}

149 Dilatancy is one of the fundamental components in modelling the stress-strain behavior of a soil. It is the ratio of the increment of plastic volumetric strain to the increment of plastic

151 deviator strain in triaxial space (Nova and Wood, 1979; Wood and Belkheir, 1994):

$$
d=\frac{d \varepsilon_{v}^{p}}{d \varepsilon_{q}^{p}}
$$

152 where $d \varepsilon_{v}=d \varepsilon_{1}+2 d \varepsilon_{3}, d \varepsilon_{q}=2\left(d \varepsilon_{1}-d \varepsilon_{3}\right) / 3$, and the superscript ' $p$ ' stands for 'plastic'. 153 The best way to investigate the dilatancy of soils is to plot dilatancy with a variation of the 154 stress ratio $\eta$

$$
\eta=q / p^{\prime}
$$

155 where $q=\sigma_{1}^{\prime}-\sigma_{3}^{\prime}$ is the deviator stress in a triaxial setting, and $p^{\prime}=\left({\sigma^{\prime}}_{1}+2 \sigma_{3}^{\prime}\right) / 3$ is the 156 effective mean stress.

157 Fig.4 (a-e) present the stress ratio-dilatancy curves at different effective confining pressures 158 for SFS+CW+RC mixtures. Note there are three stress ratios of interest for the stress ratiodilatancy curves, i.e. $\eta_{P T S}, \eta_{\text {peak }}$, and $\eta_{C S}$ where PTS stands for the phase transformation state, 'peak' refers to the peak deviator stress state, and CS means the critical state. Thus the stress ratio at these three conditions $\eta_{P T S, p e a k, C S}$ can be determined by:

$$
\eta_{P T S, p e a k, C S}=\frac{q_{P T S, p e a k, C S}}{p_{P T S, p e a k, C S}^{\prime}}
$$


162

163

164

165

166

167

168

Where $q_{P T S, p e a k, C S}$ is the deviator stress at the phase transformation state, the peak deviator stress state, or the critical state; and $p_{P T S, p e a k, C S}^{\prime}$ is the effective mean stress at the phase transformation state, the peak deviator stress state, or the critical state. Moreover, $\eta_{C S}$ can also be written as $M_{C S}$.

At the phase transformation state $d=0$, the volumetric strain $\varepsilon_{v}$ reaches its minimum value and the specimen changes from contraction to dilatancy. At the peak deviator stress state, the stress ratio $\eta$ and the deviator stress reach their peak, and at the critical state $\eta_{C S}=M_{c S}$, $d=0$, and $d \eta=0$. Note that $\eta_{\text {peak }}$ and $\eta_{P T S}$ decrease as the effective confining pressure increases, and all the SFS+CW+RC specimens experience a hook after the peak deviator stress state. It is observed that only the dilatancy of SFS+CW+RC mixtures with $0 \%$ and $10 \%$ $\mathrm{RC}$ can reach zero after the peak deviator stress state (Fig.4 a \& b), but as more RC are included $d=0$ is harder to reach, whereas the trend of dilatancy indicates it is still possible to achieve zero beyond the $25 \%$ of axial strain.

Fig.4 (f \& g) show the stress ratio-dilatancy curves of the waste mixtures with different $R_{b}$ (\%), which proves that $R_{b}$ has a significant influence on the dilatancy of waste mixtures. At the same effective confining pressure, the peak stress ratio $\eta_{\text {peak }}$ decreases as the $R_{b}$ increases due to the low shear strength. As expected, dilatancy at $\eta_{\text {peak }}$ decreases with the inclusion of RC because rubber materials are highly compressible, however the initial slope of the dilatancy curve is similar regardless of amount of RC.

\section{The critical state of $S F S+C W+R C$ mixtures}

The critical state (CS) of conventional soils is when it reaches a condition where $\frac{d q}{d \varepsilon_{q}}=\frac{d p}{d \varepsilon_{q}}=$ $\frac{d \varepsilon_{v}}{d \varepsilon_{q}}=0$; this means that once CS arrives, there will be no further changes in the deviator stress and mean effective stress upon further straining the soil, and dilatancy also reaches 
zero. To investigate the critical state of SFS $+\mathrm{CW}+\mathrm{RC}$ mixtures, triaxial tests were carried out

to the maximum axial strain possible in the apparatus; it was generally around $25 \%$.

187 Fu et al. (2017) investigated the effect of rubber particle type on the critical state of sandrubber mixtures, and the test results showed that with low rubber content (10\%) granulated rubber-sand mixtures can reach critical state within $25 \%$ of $\varepsilon_{1}$, while rubber buffing fibressand mixtures can only reach critical state at very large axial strain (i.e. $>40 \%$ ). Mashiri et al. (2015) found that mixtures of tire chips-sand with large amounts of rubber chips cannot reach CS, even when $\varepsilon_{1}=25 \%$, because tire chips keep on deforming until the end of the test (Youwai and Bergado, 2003). However, RC is much smaller than tire chips, and when they are compressed they will be surrounded by rigid particles (i.e. SFS and CW) which may prevent further deformation of RC particles. In this study, the SFS+CW+RC mixtures with lower $R_{b}(0 \%$ and $10 \%)$ can directly achieve CS, whereas those with higher $R_{b}(20-40 \%)$ cannot reach CS by the test, albeit it is still possible to reach CS beyond $\varepsilon_{1}=25 \%$ (e.g. Fig. 4 c-e). Therefore, based on $R_{b}(\%)$, two ways for determining the CS for SFS+CW+RC mixtures are suggested:

(1) With lesser $R_{b}(0 \%$ and $10 \%)$, the CS of the SFS+CW+RC mixtures can be obtained by comparing the curves in the $\varepsilon_{q}-\eta, \varepsilon_{q}-\varepsilon_{v}$, and $d-\eta$ spaces. An example of an $\mathrm{SFS}+\mathrm{CW}+\mathrm{RC}$ mixture with $10 \% \mathrm{RC}$ at $\sigma_{3}^{\prime}=10 \mathrm{kPa}$ is shown in Fig.5 (a \& b); here dilatancy decreases after $\eta_{\text {peak }}$ and reaches zero when the stress ratio $\eta=2.01$ (Fig.5b). Meanwhile, the increment of $\frac{q}{p^{\prime}}-\varepsilon_{q}$ and $\varepsilon_{v}-\varepsilon_{q}$ curves also reaches zero at the same point (Fig.5a), so the critical state of the SFS+CW+RC mixture with $10 \%$ $\mathrm{RC}$ is now determined $\left(M_{C S}=2.01\right)$.

(2) The CS of mixtures with larger $R_{b}(20 \%, 30 \%$ and $40 \%)$, can be determined by extrapolating the curves to the most probable value; Carrera et al. (2011), Indraratna 
et al. (2014), and Modoni et al. (2011) also used this method. Fig.5 (c \& d) show an example of an SFS+CW+RC mixture with $R_{b}=30 \%$ at $\sigma_{3}^{\prime}=40 \mathrm{kPa}$. Here the are obtained, and thus $M_{C S}=1.61$ has been determined.

215

The value of $M_{c s}$ and the critical void ratio $e_{c s}$ of the waste mixtures are shown in Table 1.

The results reveal that $M_{c s}$ is no longer a constant for $\mathrm{SFS}+\mathrm{CW}+\mathrm{RC}$ mixtures since it depends on the effective confining pressure and $R_{b}(\%)$. In the $e_{c s}-\ln p^{\prime}{ }_{c s}$ space a linear 218 relationship was established for the critical state line of the SFS+CW+RC mixtures (Fig.6a):

$$
e_{c s}=\Gamma-\lambda \ln p_{c s}^{\prime}
$$

219

where $\Gamma$ is the void ratio at $p_{c s}^{\prime}=1 k P a$, and $\lambda$ is the gradient of the critical state line in $e-\ln p^{\prime}$ space. It is worthy to note that the critical state lines in $e-\ln p^{\prime}$ space rotate clockwise as $R_{b}(\%)$ increases, thus indicating that $\Gamma$ and $\lambda$ are parameters associated with $R_{b}$ (\%) (Fig.6a). As expected, Fig.6 (b) shows that $\Gamma$ and $\lambda$ are in a linear relationship with $R_{b}$ (\%):

$$
\begin{aligned}
& \Gamma^{*}=\Gamma_{1}+\Gamma_{2} R_{b} \\
& \lambda^{*}=\lambda_{1}+\lambda_{2} R_{b}
\end{aligned}
$$

where $\Gamma^{*}$ and $\lambda^{*}$ are the critical state parameters modified $R_{b} . \Gamma_{1}, \Gamma_{2}, \lambda_{1}$, and $\lambda_{2}$ are the calibration parameters calculated by the test data of Group A (waste mixtures with $0 \%, 10 \%$, and $30 \% \mathrm{RC}$ ), and test data form Group B (waste mixtures with $20 \%$ and $40 \% \mathrm{RC}$ ) that were set separately and used for validation. There is a good agreement between the proposed empirical equations and the test data from Group B (Fig.6b). 


\section{Energy absorbing property of $S F S+C W+R C$ mixtures}

230 The energy absorbing property of SFS+CW+RC mixtures is captured by examining the total work $W_{\text {total }}$ until the specimen achieved its point of failure. Here failure is taken to correspond to the peak deviator stress attained, which is in the same way as Zornberg et al.

233

234 (2004) for Sand-tire shred mixtures. In general, the work given to a unit volume of SFS+CW+RC mixtures upon the applied stresses is partly consumed by friction due to particles rearrangement, and partly used by particles deformation. As the deformation of the rigid particles (i.e. SFS and CW) is negligible, the energy consumed by particle deformation is only occurred to RC. This also explains why rubber materials have high energy absorbing capacity (Zheng and Sutter, 2000). Two components are distinguished in the total work, i.e. volumetric and deviatoric, and by decoupling these two components, the increment of $\mathrm{W}$ applied onto the sample can be determined (Modoni et al., 2011)

$$
d W_{\text {total }}=p^{\prime} d \varepsilon_{v}+q d \varepsilon_{q}
$$

The value of $W_{\text {total }}$ of the mixtures is shown in Table 1 . It is worthy to note that $W_{\text {total }}$ is a parameter that incorporates the effects of $R_{b}$ and the applied stress, and it increases as $R_{b}$ and the confining stress increases. As mentioned above, $M_{C S}$ is not a constant as it changes with confining stress and $R_{b}$. Therefore, $W_{\text {total }}$ may be a good parameter to reflect the changes of $M_{C S}$. Chavez and Alonso (2003) proposed a model for rockfill using the plastic work to capture the changes of $M_{c s}$ due to suction and confining stress. However, in this study as the elastic deformation generated by RC cannot be neglected, and thus the total work $W_{\text {total }}$ is used to reflect the changes of $M_{c s}$ due to confining stress and $R_{b}$. By connecting $W_{\text {total }}$ to $M_{C S}$, an empirical relationship is established using group A data (Fig.7). In this way, the energy absorbing property of RC is translated through $W_{\text {total }}$. The equation is then validated by using group B data (independent set of data): 


$$
M_{c S}^{*}=M_{0} *\left(\frac{W_{t o t a l}}{W_{0}}\right)^{\alpha}
$$

252 where $M_{0}$ is the critical stress ratio when $W_{\text {total }}=1 \mathrm{kPa}, \alpha$ is a regression coefficient, and $253 W_{0}=1 \mathrm{kPa}$ is used to keep the unit of both side of the equation the same. It is evident that 254 Equation (8) has a good correlation with independent set of data (Group B).

\section{Dilatancy Model and Simulation for SFS+CW+RC Mixtures}

256 The dilatancy of soil is usually related to the state of soil which corresponds to its density and

257 pressures. Been and Jefferies (1985) proposed a state parameter $\psi$ to capture the influence of 258 the density and stress on the deformation of soils, where this state parameter is defined as the 259 difference between the current void ratio and the critical void ratio at the same pressure:

$$
\psi=e-e_{c s}
$$

260

261

Fig. 8 shows the definition of the state parameter. All the specimens of waste mixtures tested in this study were in a dense state after consolidation, so only $\psi<0$ appeared. After consolidation at the same effective confining pressure, the initial void ratio of the $\mathrm{SFS}+\mathrm{CW}+\mathrm{RC}$ mixtures with a greater $R_{b}(\%)$ is smaller than those with less $R_{b}(\%)$, thus proving the high compressibility of rubber materials. As mentioned previously, the critical void ratio of the waste mixtures is related to $R_{b}(\%)$, therefore the state parameter $\psi$ can be modified as:

$$
\psi^{*}=e-\left(\Gamma^{*}-\lambda^{*} \ln p_{C S}^{\prime}\right)
$$

Following, Li and Dafalias (2000), the dilatancy of soil $d$ associated with the state parameter, and is expressed as:

$$
d=\frac{d \varepsilon_{v}^{p}}{d \varepsilon_{q}^{p}}=d_{0}\left(e^{m \psi^{*}}-\frac{\eta}{M_{C S}^{*}}\right)
$$


where $d_{0}$ and $m$ are two material parameters, $M_{c s}^{*}$ is the critical stress ratio modified with $W_{\text {total }}$, and $\psi^{*}$ is the state parameter modified with $R_{b}(\%)$.

Parameter $m$ can be computed based on the phase transformation state (PTS), i.e., $d=0$, $\psi^{*}=\psi_{P T S}^{*}$, and $\eta=\eta_{P T S}$. Hence,

$$
m=\frac{1}{\psi_{P T S}^{*}} \ln \left(\frac{\eta_{P T S}}{M_{C S}^{*}}\right)
$$

273 The dilatancy parameter $d_{0}$ can be calculated at the peak deviator point, i.e., $d=d_{\text {peak }}$, 274 $\psi^{*}=\psi_{\text {peak }}^{*}$, and $\eta=\eta_{\text {peak }}$. Thus,

$$
d_{0}=\frac{d_{\text {peak }}}{\left(e^{m \psi_{\text {peak }}^{*}}-\frac{\eta_{\text {peak }}}{M_{C S}^{*}}\right)}
$$

275 The value of the dilatancy parameters is listed in Table 2. The parameters from Group A were 276 calculated from the tests data, and parameters for group B were calculated based on

277 Equations (8 and 10). Fig.9 (a-c) illustrate the dilatancy model predictions for SFS+CW+RC mixtures with $10 \%, 30 \%$, and $40 \% \mathrm{RC}$ at different effective confining pressures. Fig.9 (d)

279 shows a comparison of the dilatancy model predictions with test results of mixtures with different $R_{b}(\%)$ at $\sigma_{3}^{\prime}=70 \mathrm{kPa}$. Note that the dilatancy model clearly captures the dilatancy of SFS+CW+RC mixtures using the parameters from Group A and Group B, thus verifying the dilatancy model for the SFS+CW+RC mixtures.

\section{Conclusions}

This paper has investigated the stress-strain behavior (with special relevance to dilatancy and the critical state) of SFS+CW+RC mixtures with SFS:CW=7:3 and different RC contents $R_{b}$ the stress-strain behavior and the critical behavior of waste mixtures such that their shear strength and dilatancy decrease as $R_{b}(\%)$ increases mainly due to the low strength and 
compressible nature of the rubber. Moreover, when $R_{b}<20 \%$, the CS of the waste mixtures can be achieved directly but when $R_{b} \geq 20 \%$, the CS could not be achieved by the test,

291 albeit the trend of $\eta-d$ curve showed that CS might be achieved beyond $\varepsilon_{1}=25 \%$; thus 292 extrapolation was used for waste mixtures with $R_{b} \geq 20 \%$. On the $e-\ln p^{\prime}$ plane, a linear relationship was proposed for the critical state line dependent on $R_{b}(\%)$. Moreover, the changing $M_{c S}$ due to $R_{b}$ and confining stress was reflected by the parameter $W_{\text {total }}$ through an empirical function, and this equation also provides a way relating the energy absorbing property with $M_{c s}$.

The dilatancy behavior of the SFS $+\mathrm{CW}+\mathrm{RC}$ mixture was predicted by a modified dilatancy function. In this function the energy absorbing property of RC was captured through the relationship of $M_{c S}^{*}$ and $W_{\text {total }}$, while the dilatancy behavior with the variation of $R_{b}(\%)$ was also translated by the modified state parameter $\psi^{*}$. This dilatancy model agrees very well with the experimental data. Although this dilatancy function was developed for the $\mathrm{SFS}+\mathrm{CW}+\mathrm{RC}$ mixture, the method used to capture the energy absorbing property of rubber materials and the way of determining the critical state parameters can be a good reference for other mixtures of rubber and soil.

\section{Notions}

$$
\begin{aligned}
\mathrm{CS} & =\text { critical state, and the critical state line, respectively; } \\
\mathrm{CW} & =\text { coal wash; } \\
d & =\text { dilatancy } \\
d_{0} & =\text { dilatancy parameter; } \\
d_{\text {peak }} & =\text { dilatancy at peak deviator stress state; } \\
d \varepsilon_{v}, d \varepsilon_{v}^{p} & =\text { total and plastic volumetric strain increment, respectively; }
\end{aligned}
$$




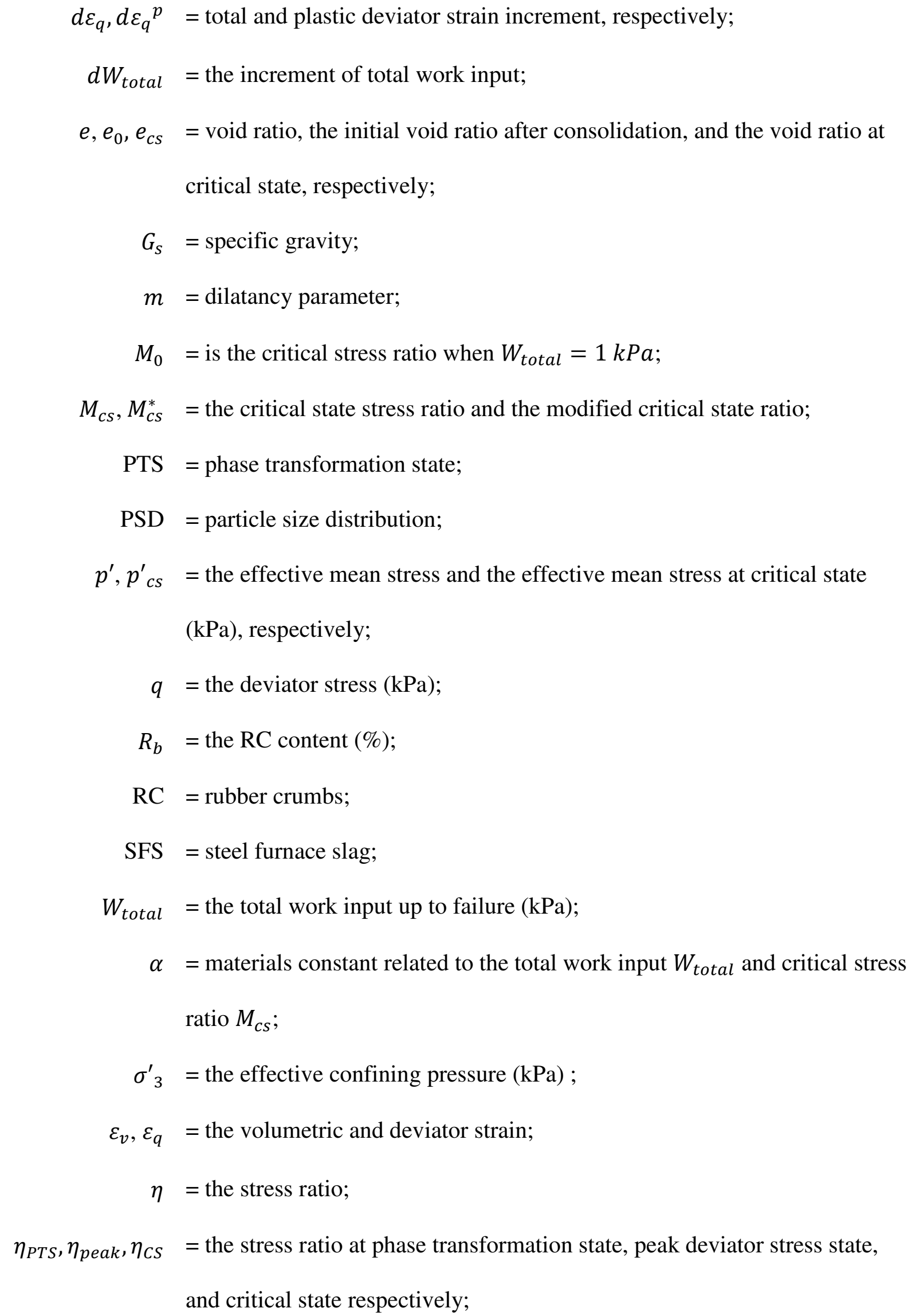




$$
\begin{aligned}
& \Gamma, \Gamma^{*}=\text { void ratio at } p^{\prime}{ }_{c s}=1 \mathrm{kPa} \text { and modified } \Gamma, \text { respectively; } \\
& \Gamma_{1}, \Gamma_{2}=\text { calibration parameters for } \Gamma ; \\
& \lambda, \lambda^{*}=\text { the gradient of the critical state line in } e-\ln p^{\prime} \text { space, and modified } \lambda, \\
& \text { respectively; } \\
& \lambda_{1}, \lambda_{2} \quad=\text { calibration parameters for } \lambda ; \\
& \psi, \psi^{*} \quad=\text { state parameter and modified state parameter, respectively; } \\
& \psi_{\text {peak }}^{*}, \psi_{P T S}^{*} \quad=\text { modified state parameter at peak deviator stress state and phase } \\
& \text { transformation state, respectively; }
\end{aligned}
$$

\section{Acknowledgements}

307 The first author would like to acknowledge the financial assistance provided by the China 308 Scholarship Council. The assistance provided by industry (ASMS and South 32) in relation to 309 the procurement of material used in this study is gratefully acknowledged. Assistance in the 310 laboratory from Mr. Richard Berndt and occasional technical feedback from A/Prof 311 Cholachat Rujikiatkamjorn and Dr Ana Heitor are also appreciated. The support from the 312 Australian Research Council Discovery Project (ARC-DP) and ARC Industry Transformation 313 Training Centre for Advanced Technologies in Rail Track Infrastructure (ITTC-Rail) is 314 gratefully appreciated.

\section{References}

316 ASTM. American Society for Testing and Materials (2011). "Standard method for 317 consolidated drained triaxial compression test for soils." D7181, West Conshohocken, PA.

318 Been, K., and Jefferies, M.J. (1985). “A state parameter for sands”. Géotechnique, 35(2), 99112. 
320 Carrera, A., Coop, M., and Lancellotta, R. (2011). "Influence of grading on the mechanical behavior of Stava tailings". Géotechnique, 61(11), 935-946.

322 Chavez, C., and Alonso, E.E. (2003). "A constitutive model for crushed granular aggregates which includes suction effects". Soil and Foundations, 43(4), 215-227.

324 Chiaro, G., Indraratna, B., Tasalloti, S.M.A., and Rujikiatkamjorn, C. (2013), "Optimisation 325 of coal wash-slag blend as a structural fill". Ground Improvement, 168(GI1), 33-44.

$326 \mathrm{Fu}, \mathrm{R}$. , Coop, M.R., and Li, X.Q. (2017). "Influence of particle type on the mechanics of 327 sand-rubber mixtures”. J. Geotech. Geoenviron. Eng., 143(9), 04017059.

Indraratna, B., Sun, Q., and Nimbalkar, S. (2014). "Observed and predicted behavior of rail ballast under monotonic loading capturing particle breakage". Can. Geotech. J., 51, 1-14. Furnace Slag, Coal Wash, and Rubber Crumbs Used as Subballast”. J. Mater. Civ. Eng., 30(1), 04017251.

Li, X.S., and Dafalias, Y.F. (2000). "Dilatancy for cohesionless soils". Géotechnique, 50(4), 449-460.

Mashiri, M., Vinod, J.S., Sheikh, M.N., and Tsang, H.H. (2015). "Shear strength and dilatancy behavior of sand-tire chip mixtures". Soil and Foundations, 55(3), 517-528. tire chip mixtures". Environmental Geotechnics, 16.00016.

339 Modoni, G., Koseki, J., and Anh Dan, L.Q. (2011). "Cyclic stress-strain response of compacted gravel". Géotechnique, 61(6), 473-485. 
341 Nova, R., and Wood, D.M. (1979). “A constitutive model for sand in triaxial compression”. Intl J. Num. Anal. Methods Geomech., 3, 255-278.

343 Rowe, P.W. (1962). "The stress-dilatancy relation for static equilibrium of an assembly of 344 particles in contact”. Proc. R. Soc., Ser. A 269, 500-527.

345 Salvatore, I., Giuseppe, M., Gabriele, C., and Erminio, S. (2015). "Predictive correlations for 346 the compaction of clean sands". Transportation Geotechnics, 4, 38-49.

347 Sheikh, M.N., Mashiri, M., Vinod, J., and Tsang, H. (2013). "Shear and Compressibility 348 Behavior of Sand-Tire Crumb Mixtures”. J. Mater. Civ. Eng., 25, 1366-1374.

349 Tasalloti, S.M.A., Indraratna, B., Rujikiatkamjorn, C., Heitor, A., and Chiaro, G. (2015). "A 350 laboratory study on the shear behavior of mixtures of coal wash and steel furnace flag as 351 potential structural fill”. ASTM Geotechnical testing journal, 38(4), 361-372.

352 Taylor, D.W. (1948). Fundamental of soil mechanics, New York, Wiley.

353 Wan, R.G., and Guo, P.J. (1998). "A simple constitutive model for granular soils: modified 354 stress-dilatancy approach”. Computers and Geotechnics, 22(2), 109-133.

355 Wang, Z.L., Dafalias, Y.F., and Shen, C.K. (1990), "Bonunding surface hypoplasticity model 356 for sand”. J. Engng Mech., 116 (5), 983-1001.

357 Wood, D.M., and Belkheir, K. (1994). "Strain softening and state parameter for sand 358 modelling". Géotechnique, 44(2), 335-339.

359 Youwai, S., and Bergado, D.T. (2003). "Strength and deformation characteristics of shredded 360 rubber tire-sand mixtures”. Canadian Geotechnical Journal, 40(2), 254-264.

361 Zheng, Y.F., and Sutter, G.K. (2000). "Dynamic properties of granulated rubber/sand 362 mixutres”. Geotechnical Testing Journal, 23(3), 338-344. 
363 Zornberg, J.G., Viratjandr, C., and Cabral, A.R. (2004). "Behavior of tire shred-sand 364 mixtures”. Canadian Geotechnical Journal, 41(2), 227-241. 


\section{Figures list}

366 Fig.1 The waste materials used in this study

367 Fig.2 Particle size distribution of the waste materials and the target PSD

368 Fig.3 Stress-strain curves for the SFS+CW+RC mixtures with different $R_{b}$ (\%): (a)-(c)

369 deviator stress versus axial strain; (d)-(f) volumetric strain versus axial strain

370 Fig.4 Stress ratio-dilatancy curve of SFS+CW+RC mixtures (a) with 0\% RC, (b) with $10 \%$

$371 \mathrm{RC}$, (c) with $20 \% \mathrm{RC}$, (d) with $30 \% \mathrm{RC}$, (e) with $40 \% \mathrm{RC}$, (f) at ${\sigma^{\prime}}_{3}=40 \mathrm{kPa},(\mathrm{g})$ at $\sigma^{\prime}{ }_{3}=$ $372 \quad 70 \mathrm{kPa}$

373 Fig.5 Determination of the critical point for waste mixtures: (a) the stress-strain curve, and (b) 374 the stress ratio-dilatancy curve for $10 \% \mathrm{RC}$ at ${\sigma^{\prime}}_{3}=10 \mathrm{kPa}$; (c) the stress-strain curve, and 375 (d) the stress ratio-dilatancy curve for $30 \% \mathrm{RC}$ at ${\sigma^{\prime}}_{3}=40 \mathrm{kPa}$

376 Fig.6 (a) $e-\ln p^{\prime}$ curves for waste mixtures at critical state; (b) the relationship of $\Gamma$ and $\lambda$ in 377 terms of $R_{b}(\%)$

378 Fig.7 The relationship of $W_{\text {total }}$ and critical stress ratio $M_{c s}$ for $\mathrm{SFS}+\mathrm{CW}+\mathrm{RC}$ mixtures

379 Fig.8 Definition of the state parameter and the critical state line for the SFS+CW+RC mixture 380 (SFS:CW=7:3) having 0\% and 30\% RC

381 Fig.9 Comparison of tests results and model predictions (stress ratio versus dilatancy) for 382 waste mixtures (SFS:CW=7:3) (a) with $10 \% \mathrm{RC}$, (b) with $30 \% \mathrm{RC}$, (c) with $40 \% \mathrm{RC}$, (d) 383 with different $R_{b}$ at $\sigma_{3}^{\prime}=70 \mathrm{kPa}$ 
385 Table 1 Critical state and total work parameters for SFS+CW+RC mixtures

386 Table 2 Model parameters for SFS+CW+RC mixtures 
Table 1 Critical state and total work parameters for SFS+CW+RC mixtures

\begin{tabular}{|c|c|c|c|c|c|c|c|c|c|c|c|}
\hline & Mixtures & $\begin{array}{l}\text { RC } \\
(\%) \\
\end{array}$ & Test No. & $\sigma_{3}^{\prime}(\mathbf{k P a})$ & $\begin{array}{l}\text { Dry density } \\
\left(\boldsymbol{k} N / \boldsymbol{m}^{3}\right)\end{array}$ & $M_{c s}$ & $\boldsymbol{e}_{\mathbf{0}}$ & $e_{c s}$ & $\boldsymbol{W}_{\text {total }}(\mathrm{kPa})$ & $\Gamma$ & $\lambda$ \\
\hline \multirow{9}{*}{$\begin{array}{c}\text { Group } \\
\text { A }\end{array}$} & \multirow{3}{*}{ SFS70+CW30 } & \multirow{3}{*}{0} & CD-1 & 10 & 19.290 & 1.94 & 0.292 & 0.395 & 2.18 & \multirow{3}{*}{0.606} & \multirow{3}{*}{0.062} \\
\hline & & & CD-2 & 40 & 19.285 & 1.804 & 0.263 & 0.328 & 9.02 & & \\
\hline & & & CD-3 & 70 & 19.298 & 1.739 & 0.231 & 0.281 & 10.79 & & \\
\hline & \multirow{3}{*}{ SFS63+CW27+RC10 } & \multirow{3}{*}{10} & CD-4 & 10 & 16.695 & 2.01 & 0.271 & 0.395 & 4.84 & \multirow{3}{*}{0.740} & \multirow{3}{*}{0.101} \\
\hline & & & CD-5 & 40 & 16.722 & 1.613 & 0.234 & 0.292 & 14.80 & & \\
\hline & & & CD-6 & 70 & 16.708 & 1.548 & 0.203 & 0.235 & 31.18 & & \\
\hline & \multirow{3}{*}{ SFS49+CW21+RC30 } & \multirow{3}{*}{30} & CD-7 & 10 & 14.735 & 2.0 & 0.271 & 0.401 & 5.46 & \multirow{3}{*}{0.94} & \multirow{3}{*}{0.159} \\
\hline & & & CD-8 & 40 & 14.742 & 1.61 & 0.220 & 0.236 & 21.93 & & \\
\hline & & & CD-9 & 70 & 14.725 & 1.48 & 0.200 & 0.162 & 38.58 & & \\
\hline \multirow{6}{*}{$\begin{array}{c}\text { Group } \\
\text { B }\end{array}$} & \multirow{3}{*}{ SFS56+CW24+RC20 } & \multirow{3}{*}{20} & CD-10 & 10 & 13.139 & 1.98 & 0.246 & 0.416 & 5.95 & \multirow{3}{*}{0.890} & \multirow{3}{*}{0.141} \\
\hline & & & CD-11 & 40 & 13.145 & 1.55 & 0.212 & 0.263 & 18.66 & & \\
\hline & & & CD-12 & 70 & 13.148 & 1.51 & 0.171 & 0.198 & 33.82 & & \\
\hline & \multirow{3}{*}{ SFS42+CW18+RC40 } & \multirow{3}{*}{40} & CD-13 & 10 & 11.789 & 1.8 & 0.250 & 0.390 & 5.81 & \multirow{3}{*}{1.012} & \multirow{3}{*}{0.183} \\
\hline & & & CD-14 & 40 & 11.785 & 1.5 & 0.208 & 0.216 & 23.28 & & \\
\hline & & & CD-15 & 70 & 11.793 & 1.43 & 0.164 & 0.148 & 42.74 & & \\
\hline
\end{tabular}

*The mixtures are expressed as SFS+CW+RC, and the numbers after SFS, CW, and RC are the percentages of steel furnace slag, coal wash, and rubber crumbs by weight. $\mathrm{CD}$ refers to consolidated drained triaxial tests, and $e_{0}$ is the initial void ratio after consolidation. Test data from

Group A were used to calibrate Equations (5, 6, and 8), and Group B was used for validation. 
Table 2 Model parameters for SFS+CW+RC mixtures

\begin{tabular}{|c|c|c|c|c|c|c|}
\hline & Mixtures & $\begin{array}{l}\mathrm{RC} \\
(\%)\end{array}$ & $\sigma_{3}^{\prime}(\mathbf{k P a})$ & $m$ & $d_{0}$ & \\
\hline \multirow{7}{*}{$\begin{array}{c}\text { Group } \\
\text { A }\end{array}$} & SFS70+CW30 & 0 & 70 & -1.30 & 3.03 & \multirow{7}{*}{$\begin{array}{c}\Gamma_{1}=0.064 \\
\Gamma_{2}=0.01 \\
\lambda_{1}=0.069 \\
\lambda_{2}=0.003 \\
\alpha=-0.117 \\
M_{0}=2.258\end{array}$} \\
\hline & \multirow{3}{*}{ SFS63+CW27+RC10 } & \multirow{3}{*}{10} & 10 & -0.46 & 2.95 & \\
\hline & & & 40 & -2.15 & 2.17 & \\
\hline & & & 70 & -2.86 & 1.83 & \\
\hline & \multirow{3}{*}{ SFS49+CW21+RC30 } & \multirow{3}{*}{30} & 10 & -0.93 & 3.80 & \\
\hline & & & 40 & -2.36 & 3.29 & \\
\hline & & & 70 & -4.16 & 2.49 & \\
\hline \multirow{6}{*}{$\begin{array}{c}\text { Group } \\
\text { B }\end{array}$} & \multirow{3}{*}{ SFS56+CW24+RC20 } & \multirow{3}{*}{20} & 10 & -0.53 & 5.12 & $M_{C S}^{*}=1.83$ \\
\hline & & & 40 & -2.98 & 2.18 & $M_{c S}^{*}=1.60$ \\
\hline & & & 70 & -5.29 & 3.19 & $M_{C S}^{*}=1.50$ \\
\hline & \multirow{3}{*}{ SFS42+CW18+RC40 } & \multirow{3}{*}{40} & 10 & -0.76 & 3.27 & $M_{C S}^{*}=1.84$ \\
\hline & & & 40 & -2.48 & 2.45 & $M_{C S}^{*}=1.53$ \\
\hline & & & 70 & -4.01 & 3.05 & $M_{C S}^{*}=1.46$ \\
\hline
\end{tabular}



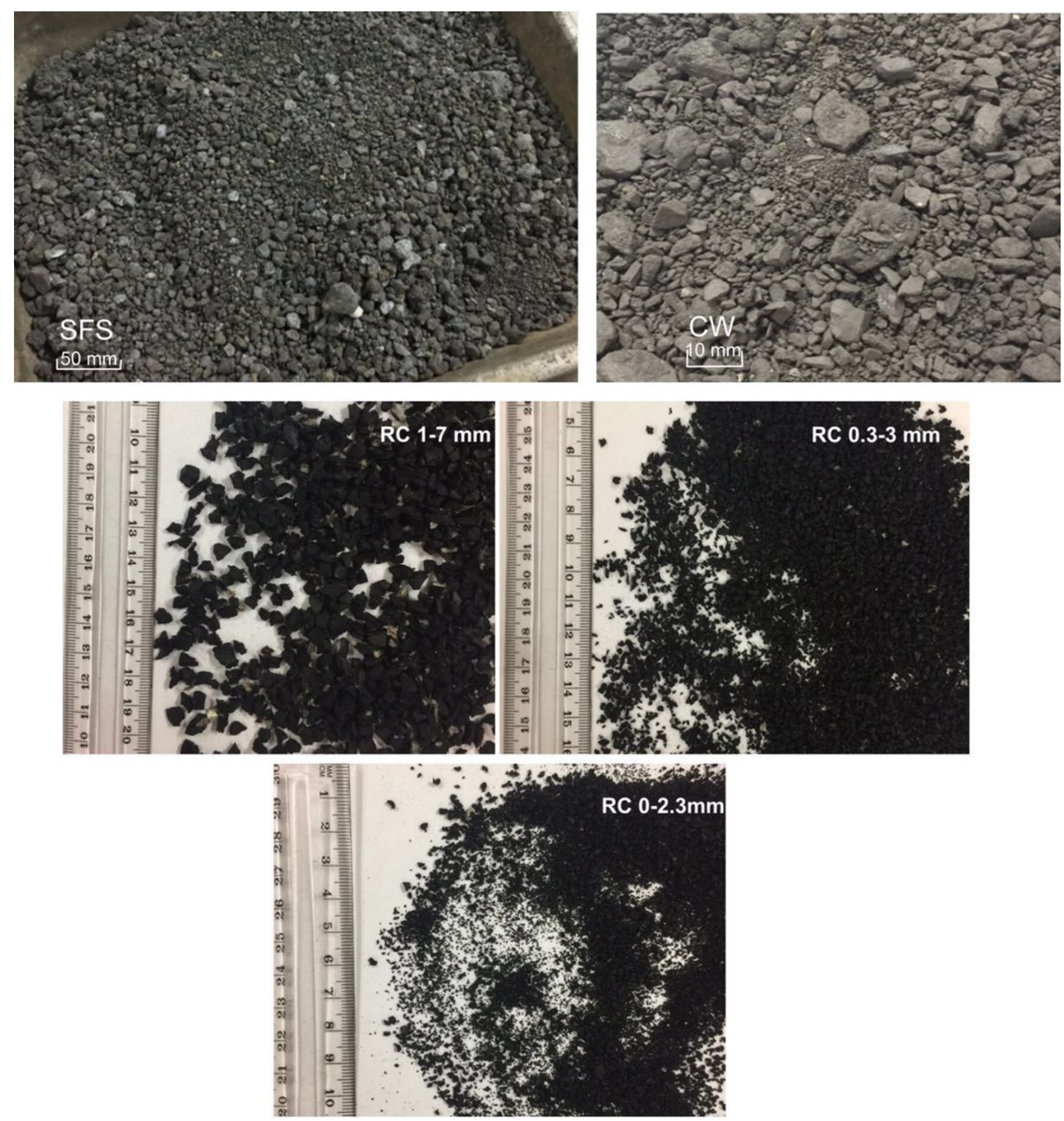


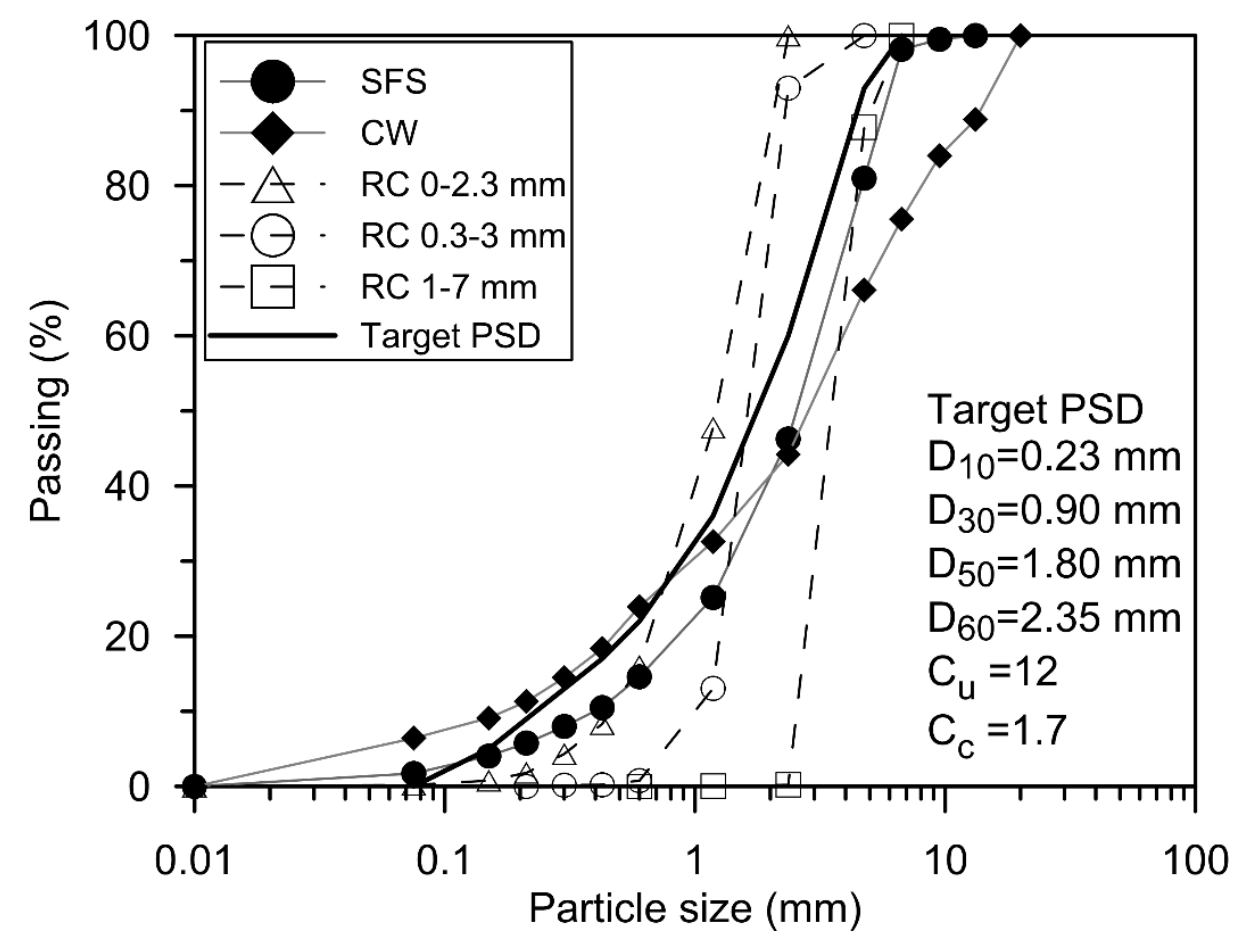

Fig.2 Particle size distribution of the waste materials and the target PSD 



401 Fig.3 Stress-strain curves for the SFS+CW+RC mixtures with different $R_{b}$ (\%): (a)-(c) deviator stress versus axial strain; (d)-(f) volumetric strain versus axial strain 

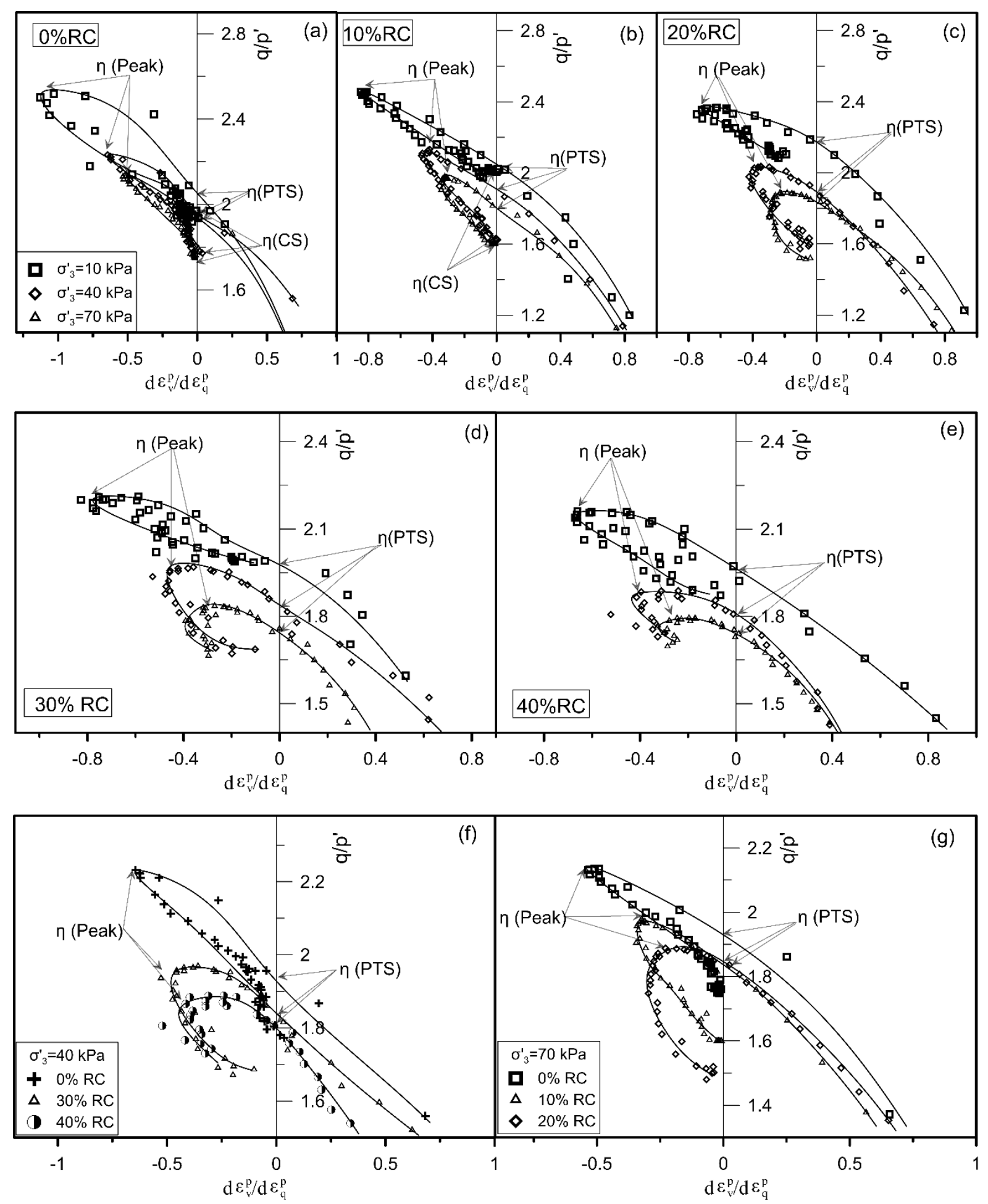

Fig.4 Stress ratio-dilatancy curve of SFS+CW+RC mixtures (a) with $0 \% \mathrm{RC}$, (b) with $10 \%$ $\mathrm{RC}$, (c) with $20 \% \mathrm{RC}$, (d) with $30 \% \mathrm{RC}$, (e) with $40 \% \mathrm{RC}$, (f) at ${\sigma^{\prime}}_{3}=40 \mathrm{kPa}$, (g) at ${\sigma^{\prime}}_{3}=$ 

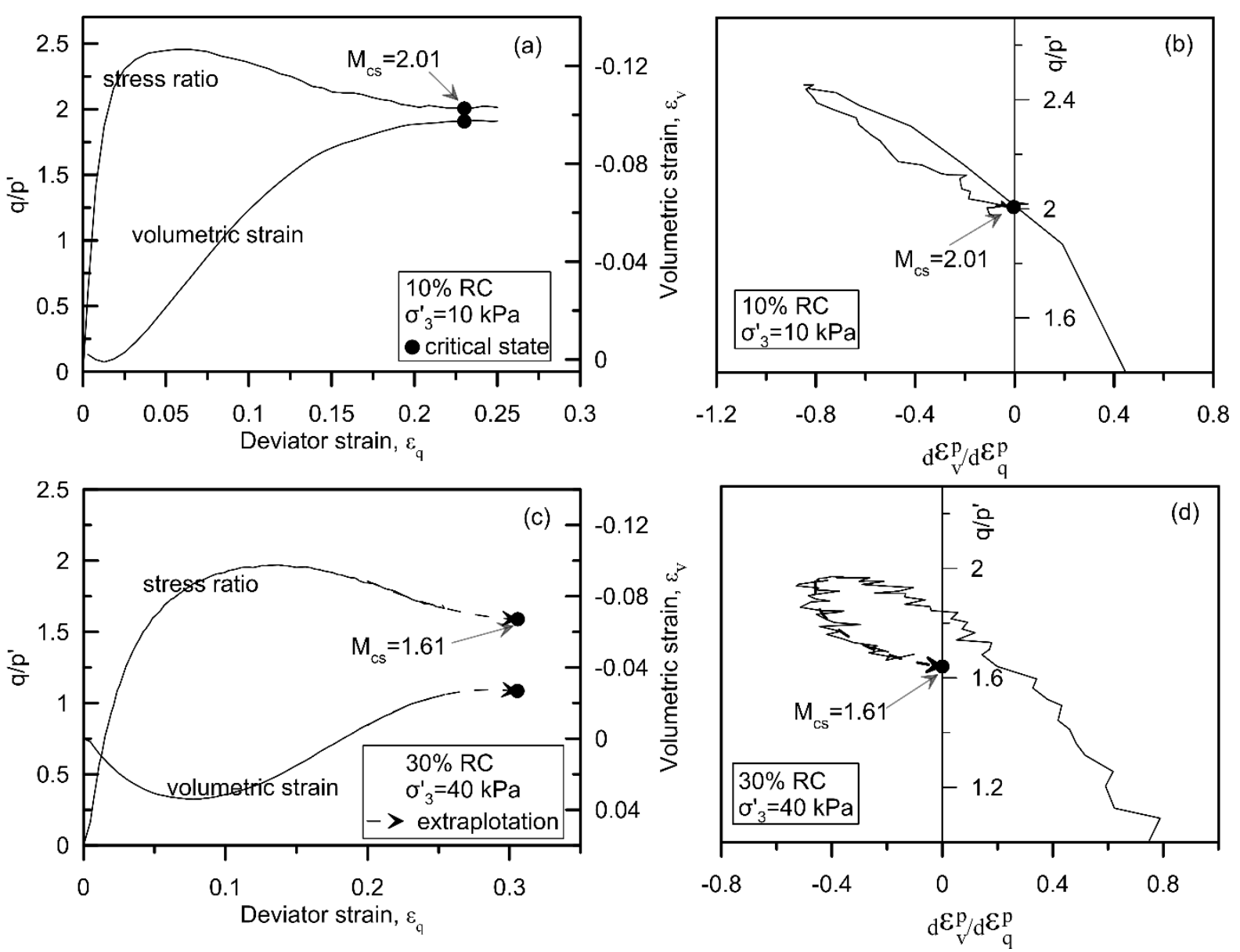

409

410 Fig.5 Determination of the critical point for waste mixtures: (a) the stress-strain curve, and (b)

411 the stress ratio-dilatancy curve for $10 \% \mathrm{RC}$ at $\sigma_{3}^{\prime}=10 \mathrm{kPa}$; (c) the stress-strain curve, and

412

(d) the stress ratio-dilatancy curve for $30 \% \mathrm{RC}$ at ${\sigma^{\prime}}_{3}=40 \mathrm{kPa}$ 

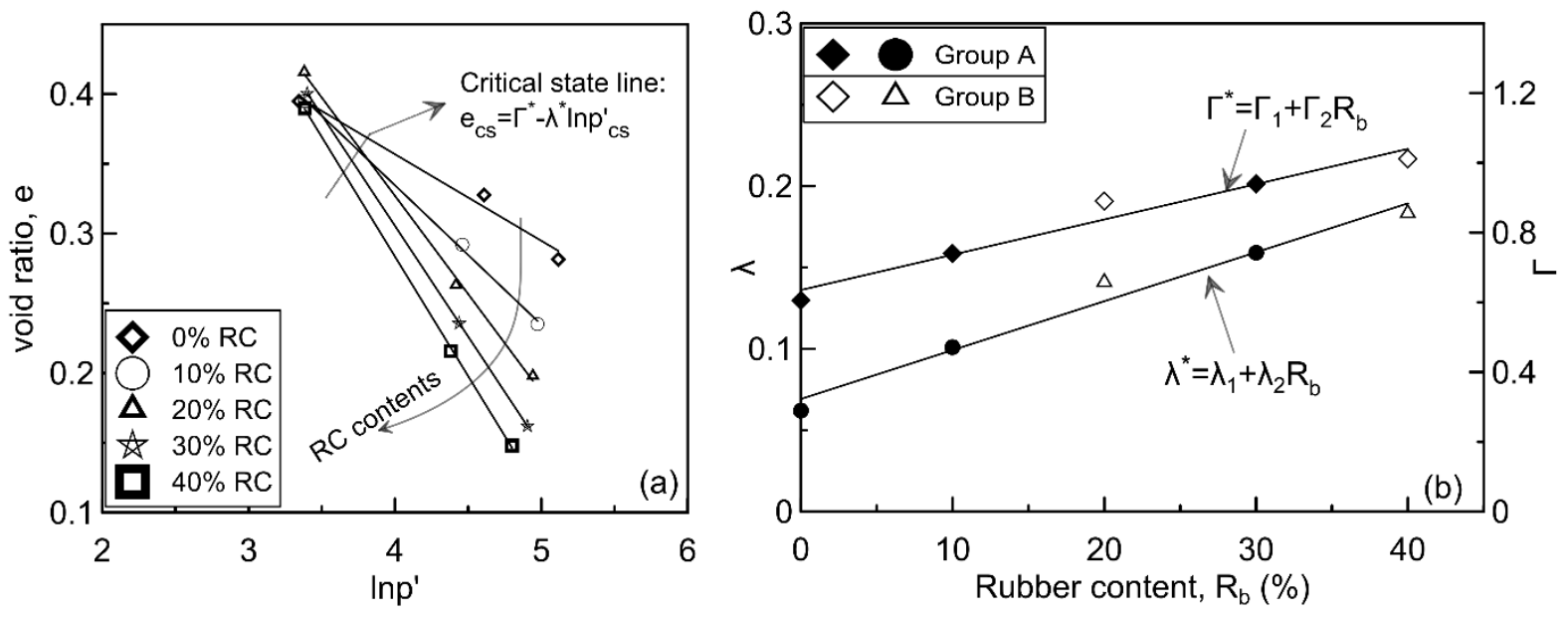

Fig.6 (a) $e-\ln p^{\prime}$ curves for waste mixtures at critical state; (b) the relationship of $\Gamma$ and $\lambda$ in

terms of $R_{b}(\%)$

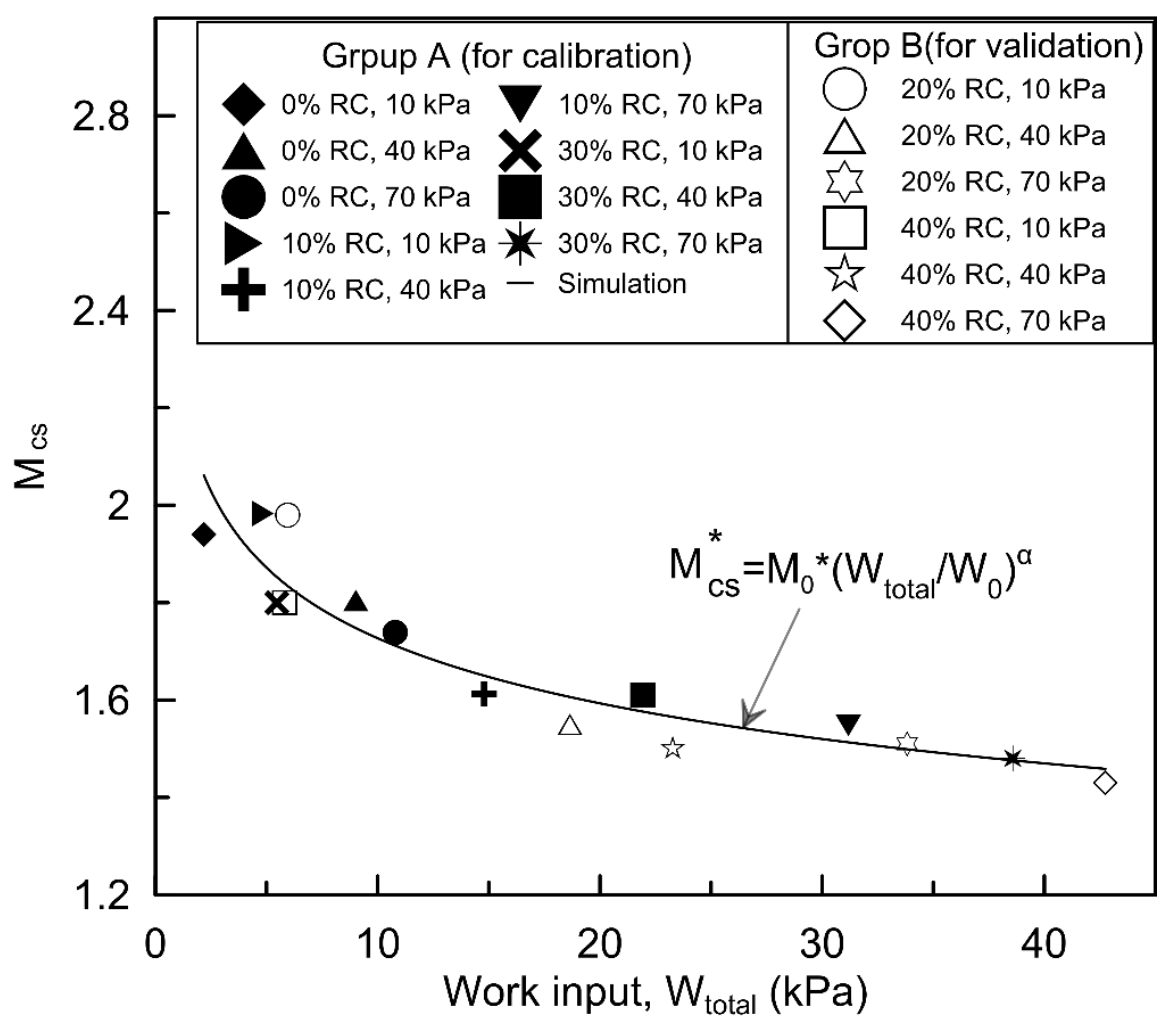

419 Fig.7 The relationship of $W_{\text {total }}$ and critical stress ratio $M_{c s}$ for SFS+CW+RC mixtures 


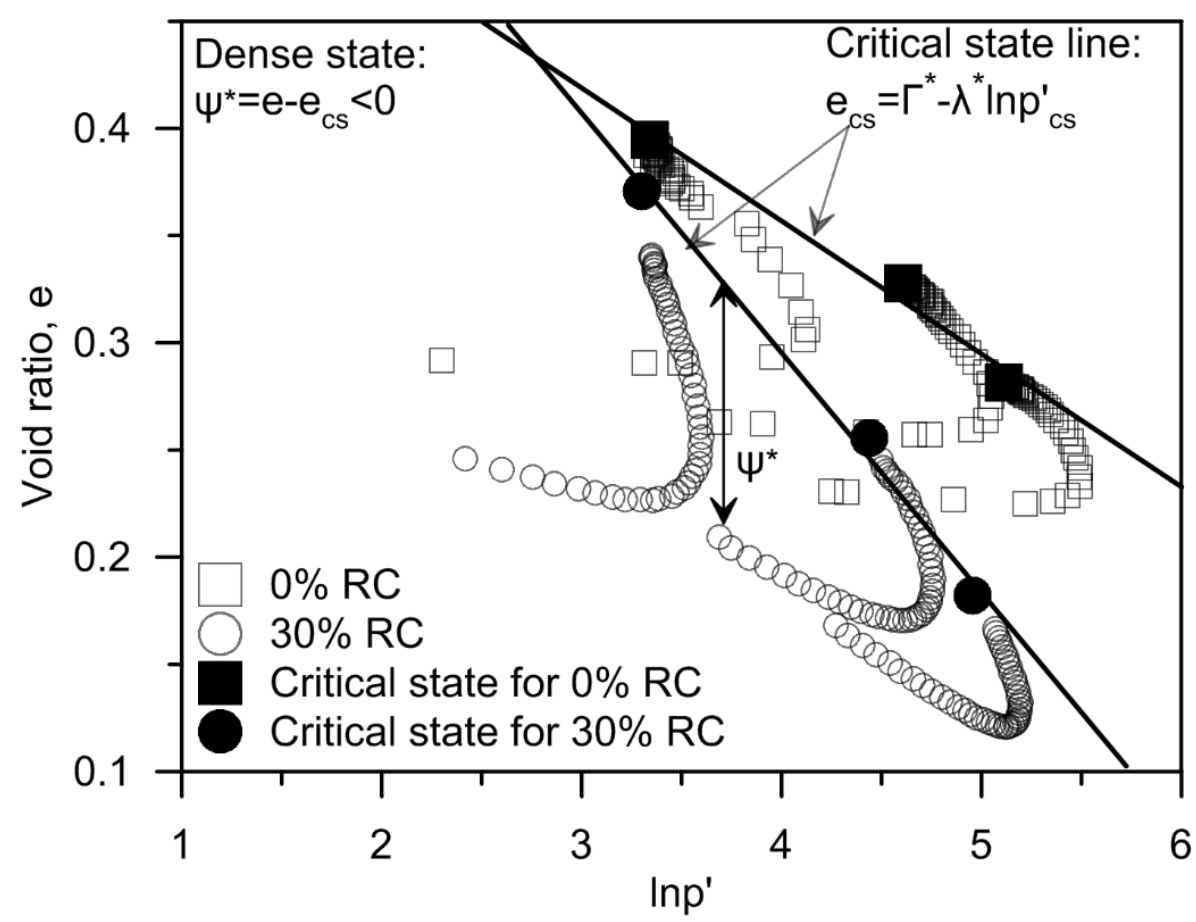

422 Fig.8 Definition of the state parameter and the critical state line for the SFS+CW+RC mixture 

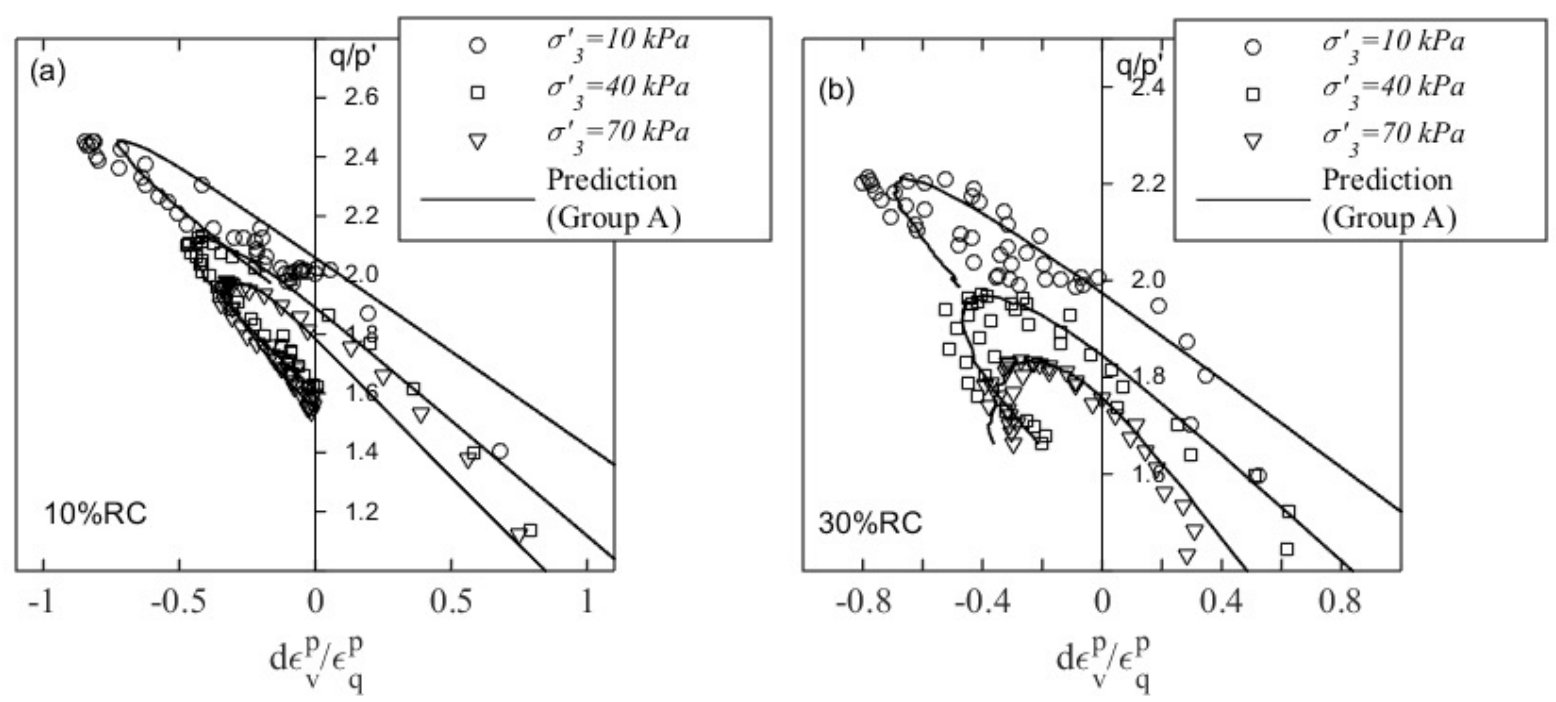

425
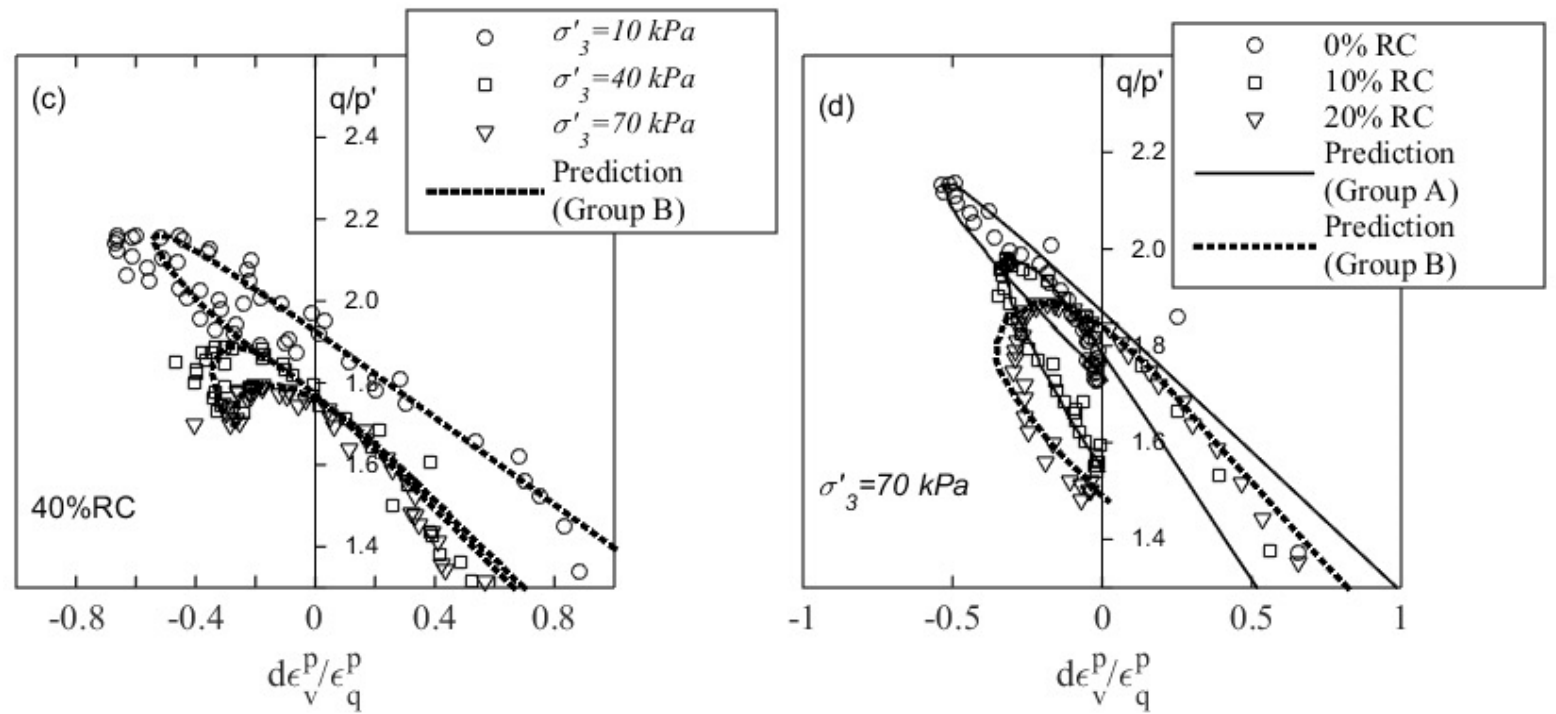

426

Fig.9 Comparison of tests results and model predictions (stress ratio versus dilatancy) for

427 waste mixtures (SFS:CW=7:3) (a) with $10 \% \mathrm{RC}$, (b) with $30 \% \mathrm{RC}$, (c) with $40 \% \mathrm{RC}$, (d) 